SLAC-PUB-8197

July 1999

\title{
Compton Scattering at the NLC and Large Extra Dimensions"
}

\author{
Hooman Davoudiasl \\ Stanford Linear Accelerator Center \\ Stanford University, Stanford, California 94309 \\ e-mail: hooman@slac.stanford.edu
}

\begin{abstract}
We study Compton scattering, $\gamma e \rightarrow \gamma e$, in the context of the recent proposal for Weak Scale Quantum Gravity (WSQG) with large extra dimensions. It is shown that, with an ultraviolet cutoff $M_{S} \sim 1 \mathrm{TeV}$ for the effective gravity theory, the cross section for this process at the Next Linear Collider (NLC) deviates from the prediction of the Standard Model significantly. Our results suggest that, for typical proposed NLC energies and luminosities, WSQG can be tested in the range $4 \mathrm{TeV} \lesssim M_{S} \lesssim 16 \mathrm{TeV}$, making $\gamma e \rightarrow \gamma e$ an important test channel.
\end{abstract}

*Work supported by the Department of Energy, contract DE-AC03-76SF00515. 


\section{Introduction}

It has recently been proposed that the fundamental scale of quantum gravity $M_{F}$ can be of the order of the weak scale $\Lambda_{w}$ if we assume that there are $n$ large spatial extra dimensions [1]. Gravitational data at macroscopic scales demand $n \geq 2$ [ and cosmological and astrophysical observations suggest that $M_{F} \gtrsim 100 \mathrm{TeV}$ for $n=$ 2 [3]. However, the data from collider experiments at present energies, as well as cosmological and astrophysical considerations for $n \geq 3$, only yield $M_{F} \gtrsim 1 \mathrm{TeV}$. Once larger center of mass energies become available at future experimental facilities, the predictions of Weak Scale Quantum Gravity (WSQG) can be tested in the TeV regime, as demonstrated by various recent works on this subject $\llbracket$ |

In this paper, we consider the possibility of testing WSQG at a future Next Linear Collider (NLC), using $\mathrm{TeV}$-scale Compton scattering $\gamma e \rightarrow \gamma e$. It has been shown that a high energy and luminosity $e^{+} e^{-}$collider can yield $\gamma$-beams comparable in energy and luminosity, using backward Compton scattering of laser photons from the $e^{ \pm}$beams [5]. We will show that given the proposed energies and luminosities of the NLC [6], and assuming the above $\gamma$-beams can be obtained, the process $\gamma e \rightarrow \gamma e$ places strong bounds on the effective mass scale of WSQG.

The present work is organized as follows. In section 2, we present the tree level Standard Model (SM) and WSQG amplitudes for $\gamma e \rightarrow \gamma e$ and the formulas for calculating the relevant cross sections at the NLC. The results of our computations are given in section 3. Section 4 contains our concluding remarks. Finally, some of the formulas used in our calculations are given in the appendix.

\section{Amplitudes and Cross Sections}

Here, we present the tree level SM and WSQG amplitudes for the process $\gamma\left(k_{1}\right) e\left(p_{1}\right) \rightarrow$ $\gamma\left(k_{2}\right) e\left(p_{2}\right)$, where $\left(k_{1}, p_{1}\right)$ are the 4 -momenta of the initial state photon and electron and $\left(k_{2}, p_{2}\right)$ are the 4-momenta of the final state photon and electron, respectively. For the rest of this work, it is assumed that the fundamental scale of gravity $M_{F} \gtrsim 1$ $\mathrm{TeV}$ and that there are $n \geq 2$ compact extra dimensions of size $R$, even though there are astrophysical and cosmological considerations that suggest $M_{F} \gtrsim 100 \mathrm{TeV}$ for

\footnotetext{
${ }^{\dagger}$ The authors of Ref. [2] propose a scenario in which $n=1$ can be allowed.
} 
$n=2$ [3]. Then, Gauss' law yields the relation [1]

$$
M_{P}^{2} \sim M_{F}^{n+2} R^{n}
$$

where $M_{P} \sim 10^{19} \mathrm{GeV}$ is the Planck mass. The exact relation among $M_{P}, M_{F}$, and $R$ is presented in the appendix and depends on the convention and the compactification manifold used. We will use the effective Lagrangian and the Feynman rules of Ref. [7].

Let $s \equiv\left(k_{1}+p_{1}\right)^{2}, t \equiv\left(k_{1}-k_{2}\right)^{2}$, and $u \equiv\left(k_{1}-p_{2}\right)^{2}$, where $k_{1}+p_{1}=k_{2}+p_{2}$. The SM contributes through the $s$ and $u$ channels with the amplitudes

$$
\mathcal{M}_{S M}^{(s)}=-\left(\frac{4 \pi \alpha}{s}\right) \varepsilon_{\mu}^{*}\left(k_{2}\right) \varepsilon_{\nu}\left(k_{1}\right) \bar{u}\left(p_{2}\right) \gamma^{\mu}\left(2 p_{1}^{\nu}+\not k_{1} \gamma^{\nu}\right) u\left(p_{1}\right)
$$

and

$$
\mathcal{M}_{S M}^{(u)}=-\left(\frac{4 \pi \alpha}{u}\right) \varepsilon_{\mu}^{*}\left(k_{2}\right) \varepsilon_{\nu}\left(k_{1}\right) \bar{u}\left(p_{2}\right) \gamma^{\nu}\left(2 p_{1}^{\mu}-\not k_{2} \gamma^{\mu}\right) u\left(p_{1}\right),
$$

respectively, where $\alpha=1 / 137$. The 4 -vector $\varepsilon^{\mu}(k)$ denotes the polarization vector for a photon of 4-momentum $k$, and $u(p)$ denotes the Dirac spinor for an electron of 4-momentum $p$.

The $t$-channel contribution of WSQG has the amplitude

$$
\begin{aligned}
\mathcal{M}_{W S Q G}^{(t)}= & -\left(\frac{\pi}{M_{S}^{4}}\right) D_{n}(t) \bar{u}\left(p_{2}\right)\left[\gamma_{\mu}\left(p_{1 \nu}+p_{2 \nu}\right)+\gamma_{\nu}\left(p_{1 \mu}+p_{2 \mu}\right)\right] u\left(p_{1}\right) \\
& \times\left[\left(k_{1} \cdot k_{2}\right) C^{\mu \nu, \alpha \beta}+D^{\mu \nu, \alpha \beta}\left(k_{1}, k_{2}\right)\right] \varepsilon_{\beta}^{*}\left(k_{2}\right) \varepsilon_{\alpha}\left(k_{1}\right),
\end{aligned}
$$

where $M_{S}$ is a momentum cutoff for the effective WSQG Lagrangian; we have taken $M_{S}=M_{F}$ here. The function $D_{n}(x)$ depends on $n$ and is given by [7]

$$
D_{n}(x) \approx \ln \left(\frac{M_{S}^{2}}{|x|}\right) \text { for } n=2
$$

and

$$
D_{n}(x) \approx\left(\frac{2}{n-2}\right) \text { for } n>2 .
$$

The expressions for $C^{\mu \nu, \lambda \sigma}$ and $D^{\mu \nu, \lambda \sigma}(k, p)$ are presented in the appendix. Note that the expressions for $D_{n}(x)$ depend on the cutoff scale $M_{S} \gg s,|t|,|u|$, introduced to regulate the divergent sum over the infinite tower of Kaluza-Klein states. This 
dependence is a result of our assumption that $M_{S}=M_{F}$. However, if $M_{S}$ is taken to be much smaller than $M_{F}$ then

$$
D_{n}(x) \rightarrow\left(\frac{M_{S}}{M_{F}}\right)^{(n+2)} D_{n}(x) \text { for } n \geq 2,
$$

resulting in a suppression 8 .

The total tree level amplitude $\mathcal{M}^{(T O T)}$ for $\gamma e \rightarrow \gamma e$, including the contributions of both the SM and WSQG, is given by

$$
\mathcal{M}^{(T O T)}=\mathcal{M}_{S M}^{(s)}+\mathcal{M}_{S M}^{(u)}+\mathcal{M}_{W S Q G}^{(t)} .
$$

We note that because of our lack of knowledge of the fundamental theory of gravity, the sign and magnitude of the lowest dimension contribution of the effective WSQG Lagrangian in Eq. (7) is undetermined. However, as long as one is only interested in an order of magnitude estimate of the size of the WSQG contribution, using $\mathcal{M}^{(T O T)}$ of Eq. (7) is reasonable.

As mentioned before, high energy and luminosity $\gamma$ beams can be achieved at the NLC, through backward Compton scattering of laser photons from the high energy $e^{ \pm}$beams [5]. The $\gamma$ beams that are obtained in this way have distributions in energy and helicity that are functions of the $\gamma$ energy and the initial polarizations of the electron beams and the laser beams. Laser beam polarization $P_{l}$ can be achieved close to $100 \%$, however, electron beam polarization $P_{e}$ is at the $90 \%$ level. We take $\left|P_{l}\right|=1$ and $\left|P_{e}\right|=0.9$ for our calculations.

Let $E_{e}$ be the electron beam energy, and $E_{\gamma}$ be the scattered $\gamma$ energy in the laboratory frame. The fraction of the beam energy taken away by the photon is then

$$
x=\frac{E_{\gamma}}{E_{e}} .
$$

We take the laser photons to have energy $E_{l}$. Then, the maximum value of $x$ is given by

$$
x_{\max }=\frac{z}{1+z},
$$

where $z=4 E_{e} E_{l} / m_{e}^{2}$, and $m_{e}$ is the electron mass. One cannot increase $x_{\max }$ simply by increasing $E_{l}$, since this makes the process less efficient because of $e^{+} e^{-}$pair production through the interactions of the laser photons and the backward scattered $\gamma$ beam. The optimal value for $z$ is given by

$$
z_{O P T}=2(1+\sqrt{2})
$$


The photon number density $f\left(x, P_{e}, P_{l}\right)$ and average helicity $\xi_{2}\left(x, P_{e}, P_{l}\right)$ are functions of $x, P_{e}, P_{l}$, and $z$, however, we always set $z=z_{O P T}$ in our calculations. We give the expressions for these two functions in the appendix.

Let $\mathcal{M}_{i j k l}, i, j, k, l= \pm$, denote the helicity amplitudes for $\gamma e \rightarrow \gamma e$, where $(i, j)$ are the helicities of the initial state $(\gamma, e)$, and $(k, l)$ are the helicities of the final state $(\gamma, e)$, respectively. We define $\left|\mathcal{M}_{i j}\right|^{2}$ by

$$
\left|\mathcal{M}_{i j}\right|^{2} \equiv \sum_{k, l}\left|\mathcal{M}_{i j k l}\right|^{2}
$$

where the summation is performed over the final state helicities. Then, for various choices of $\left(P_{e_{1}}, P_{l_{1}}\right)$ of the $\gamma$ beam and $P_{e_{2}}$ of the electron beam, the differential cross section $d \sigma / d \Omega$ is given by

$$
\frac{d \sigma}{d \Omega}=\frac{1}{(8 \pi)^{2}} \int \frac{d x f(x)}{x s_{e e}}\left[\left(\frac{1+P_{e_{2}} \xi_{2}(x)}{2}\right)\left|\mathcal{M}_{++}\right|^{2}+\left(\frac{1-P_{e_{2}} \xi_{2}(x)}{2}\right)\left|\mathcal{M}_{+-}\right|^{2}\right]
$$

where $s_{e e}=4 E_{e}^{2}$. Different choices of $\left(P_{e_{1}}, P_{l_{1}}\right)$, in $\left(f(x), \xi_{2}(x)\right)$, and $P_{e_{2}}$ yield different polarization cross sections. We note that the expressions for $\left|\mathcal{M}_{++}\right|^{2}$ and $\left|\mathcal{M}_{+-}\right|^{2}$ are actually functions of the $\gamma e$ center of mass energy squared $\hat{s}=x s_{e e}$, and the center of mass scattering angle $\theta_{c m}$. We also have $t \rightarrow \hat{t}$ and $u \rightarrow \hat{u}$, where $\hat{t}=$ $-(\hat{s} / 2)\left(1-\cos \theta_{c m}\right)$ and $\hat{u}=-(\hat{s} / 2)\left(1+\cos \theta_{c m}\right)$. In the following calculations, we use Eq. (12) and the cuts

$$
\theta_{c m} \in[\pi / 6,5 \pi / 6] ; x \in\left[0.1, x_{\max }\right]
$$

to obtain the cross sections.

\section{Results}

In this section, we present our numerical results for the expected size of the WSQG effects at the NLC. However, before discussing the results, we would like to make a few remarks regarding our calculations. First of all, as mentioned before, we have assumed $M_{S}=M_{F}$ in our calculations. The effects of departure from this assumption are given in Eq. (6). Secondly, the only dependence on the number of extra dimensions $n$ in our computations comes from Eq. (5). We only distinguish between the cases with $n=2$ and $n>2$. In the case with $n=2$, in the limit $M_{S}^{2} \gg s$, the WSQG amplitude 
is enhanced logarithmically compared to the case with $n>2$. In our computations, for $n>2$, we have $\ln \left(M_{S}^{2} / \hat{t}\right)>2 /(n-2)$ over most of the parameter space considered. We choose $n=4$ as a representative value for $n>2$; other choices result in a rescaling of the effective value of $M_{S}$.

The SM + WSQG cross sections for $M_{S}=2 \mathrm{TeV}, n=4$, and $\sqrt{s_{e e}} \in[500,1500]$ $\mathrm{GeV}$, with four independent choices of the initial polarization $\left(P_{e_{1}}, P_{l_{1}}, P_{e_{2}}\right)$ are given in Fig. (11). The largest high energy cross section is that with the polarization $(+,-,+)$, and we will use this polarization for the rest of our calculations. In Fig. (21), we compare the SM + WSQG cross sections for $M_{S}=2 \mathrm{TeV}$ and $n=2,4$ with the SM cross section, over the range $\sqrt{s_{e e}} \in[500,1500] \mathrm{GeV}$. We see that, in Fig. (2), the cross section with $n=2$ is larger than the cross section with $n=4$, because of the aforementioned logarithmic enhancement.

The differential cross sections $d \sigma / d\left(\cos \theta_{c m}\right)$ at $\sqrt{s_{e e}}=1500 \mathrm{GeV}$ for SM, and SM + WSQG, with $M_{S}=2 \mathrm{TeV}$ and $n=2,4$, are presented in Fig. (3). We see that at this value of $\sqrt{s_{e e}}$, the SM + WSQG angular distributions for $\gamma e \rightarrow \gamma e$ are very different from the prediction of the SM. The SM + WSQG differential cross section with $n=2$ is enhanced in the forward direction, since $\ln \left(M_{S}^{2} / \hat{t}\right) \rightarrow \infty$ as $\theta_{c m} \rightarrow 0$.

The $M_{S}$ reach at the NLC with center of mass energies of $500 \mathrm{GeV}, 1000 \mathrm{GeV}$, and $1500 \mathrm{GeV}$ are shown in Fig. (4). To obtain the reach, we have used the $\chi^{2}\left(M_{S}\right)$ variable given by

$$
\chi^{2}\left(M_{S}\right)=\left(\frac{L}{\sigma_{S M}}\right)\left[\sigma_{S M}-\sigma\left(M_{S}\right)\right]^{2},
$$

where $L$ is the luminosity, $\sigma_{S M}$ is the SM cross section, and $\sigma\left(M_{S}\right)$ is the $\mathrm{SM}+$ WSQG cross section as a function of $M_{S}$. We have taken $L=100 \mathrm{fb}^{-1}$ per year for our calculations, and the initial polarization $(+,-,+)$ has been used for all the cross sections. To get the reach, we demand $\chi^{2}\left(M_{S}\right) \geq 2.706$, corresponding to a one-sided 95\% confidence level. The smallest reach in Fig. (田) is about $4 \mathrm{TeV}$ for $n=4$ and $\sqrt{s_{e e}}=500 \mathrm{GeV}$ and the largest reach is about $16 \mathrm{TeV}$ for $n=2$ and $\sqrt{s_{e e}}=1500 \mathrm{GeV}$. Note that the reach for $n=2$ at $\sqrt{s_{e e}}=500 \mathrm{GeV}$ is about 7 $\mathrm{TeV}$ or approximately $14 \sqrt{s_{e e}}$. According to Eq. (14), the reach can be improved by increasing the luminosity $L$. However, we have checked that using $L=200 \mathrm{fb}^{-1}$ per year does not improve the reach significantly. 


\section{Concluding Remarks}

In this work, we have shown that the NLC with the photon collider option can be effectively used to constrain theories of weak scale quantum gravity by measuring the scattering process $\gamma e \rightarrow \gamma e$ at $\mathrm{TeV}$ energies. The size of the expected effect depends on the choice of the electron and laser polarizations. The results of this paper suggest that studying $\gamma e \rightarrow \gamma e$ at the NLC, operating at $\sqrt{s_{e e}} \in[500,1500]$ $\mathrm{GeV}$ and $L=100 \mathrm{fb}^{-1}$ per year, can constrain the scale $M_{S}$ at which quantum gravity becomes important, over the range $4 \mathrm{TeV} \lesssim M_{S} \lesssim 16 \mathrm{TeV}$. This makes $\gamma e \rightarrow \gamma e$ one of the most promising discovery channels for weak scale quantum gravity at the NLC.

\section{Acknowledgements}

It is a pleasure to thank J. Hewett, M. Peskin, J. Rathsman, and T. Rizzo for various comments and conversations.

\section{Appendix}

In this paper, we have assumed that the fundamental mass scale $M_{F}$ of gravity and the size $R$ of the $n$ extra dimensions are related by [7]

$$
\kappa^{2} R^{n}=16 \pi(4 \pi)^{n / 2} \Gamma(n / 2) M_{F}^{-(n+2)},
$$

where $\kappa=\sqrt{16 \pi G_{N}} ; G_{N}$ is the four dimensional Newton constant and $\Gamma$ represents the Gamma-function.

The expressions for $C_{\mu \nu, \lambda \sigma}$ and $D_{\mu \nu, \lambda \sigma}(k, p)$, used in Eq. (4), are given by [7]

$$
C_{\mu \nu, \lambda \sigma}=\eta_{\mu \lambda} \eta_{\nu \sigma}+\eta_{\mu \sigma} \eta_{\nu \lambda}-\eta_{\mu \nu} \eta_{\lambda \sigma}
$$

and

$$
D_{\mu \nu, \lambda \sigma}(k, p)=\eta_{\mu \nu} k_{\sigma} p_{\lambda}-\left[\eta_{\mu \sigma} k_{\nu} p_{\lambda}+\eta_{\mu \lambda} k_{\sigma} p_{\nu}-\eta_{\lambda \sigma} k_{\mu} p_{\nu}+(\mu \leftrightarrow \nu)\right]
$$

respectively, where $\eta_{\mu \nu}$ is the Minkowski metric tensor. 
Let $P_{e}$ and $P_{l}$ be the polarizations of the electron beam and the laser beam, respectively. We define the function $C(x)[5]$ by

$$
C(x) \equiv \frac{1}{1-x}+(1-x)-4 r(1-r)-P_{e} P_{l} r z(2 r-1)(2-x),
$$

where $r \equiv x /[z(1-x)]$. Then, the photon number density $f\left(x, P_{e}, P_{l} ; z\right)$ is given by

$$
f\left(x, P_{e}, P_{l} ; z\right)=\left(\frac{2 \pi \alpha^{2}}{m_{e}^{2} z \sigma_{C}}\right) C(x),
$$

where

$$
\begin{aligned}
& \sigma_{C}=\left(\frac{2 \pi \alpha^{2}}{m_{e}^{2} z}\right)\left[\left(1-\frac{4}{z}-\frac{8}{z^{2}}\right) \ln (z+1)+\frac{1}{2}+\frac{8}{z}-\frac{1}{2(z+1)^{2}}\right] \\
& +P_{e} P_{l}\left(\frac{2 \pi \alpha^{2}}{m_{e}^{2} z}\right)\left[\left(1+\frac{2}{z}\right) \ln (z+1)-\frac{5}{2}+\frac{1}{z+1}-\frac{1}{2(z+1)^{2}}\right] .
\end{aligned}
$$

The average helicity $\xi_{2}\left(x, P_{e}, P_{l} ; z\right)$ is given by

$$
\xi_{2}\left(x, P_{e}, P_{l} ; z\right)=\frac{1}{C(x)}\left\{P_{e}\left[\frac{x}{1-x}+x(2 r-1)^{2}\right]-P_{l}(2 r-1)\left(1-x+\frac{1}{1-x}\right)\right\} .
$$

\section{$\left(P_{e_{1}}, P_{l_{1}}, P_{e_{2}}\right)$ Polarization Cross Sections}

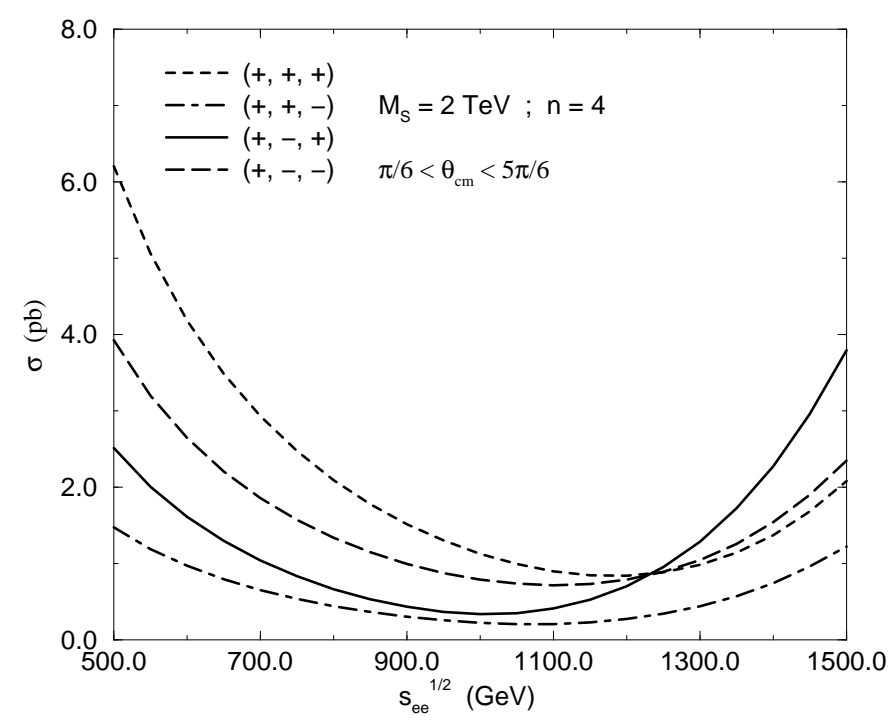

Figure 1: SM + WSQG cross sections with four independent initial electron and laser beam polarizations. Here, $M_{S}=2 \mathrm{TeV}$ and $n=4$. 


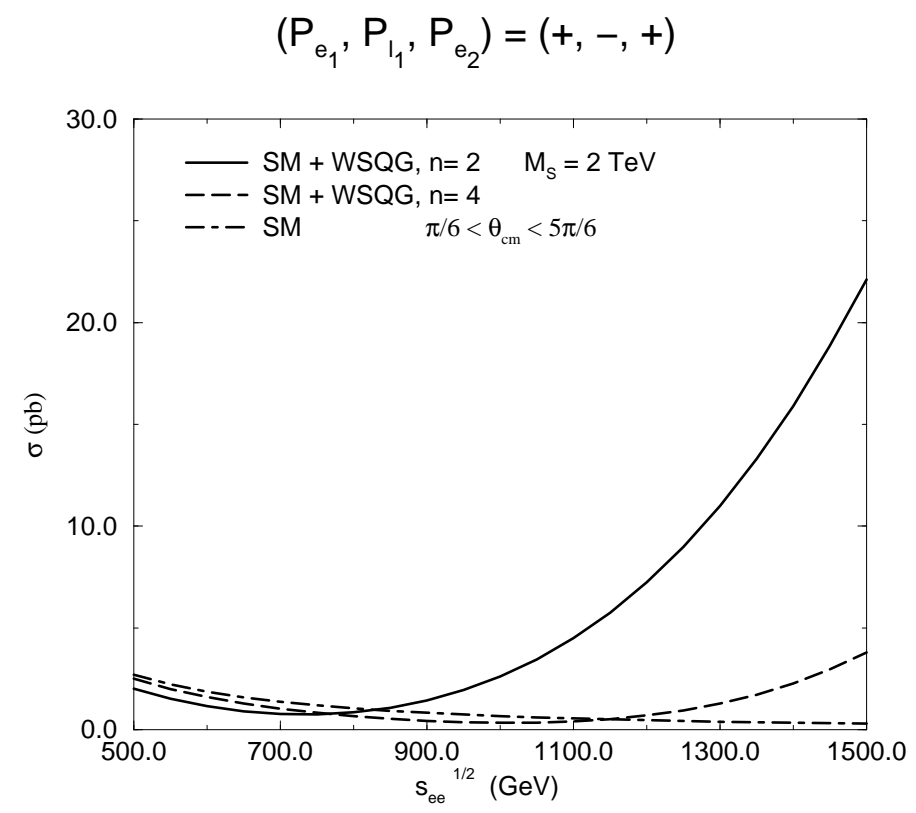

Figure 2: SM + WSQG and SM cross sections for the $(+,-,+)$ polarization. Here, $M_{S}=2 \mathrm{TeV}$ and $n=2,4$, for the WSQG contributions.

\section{Differential Cross Sections}

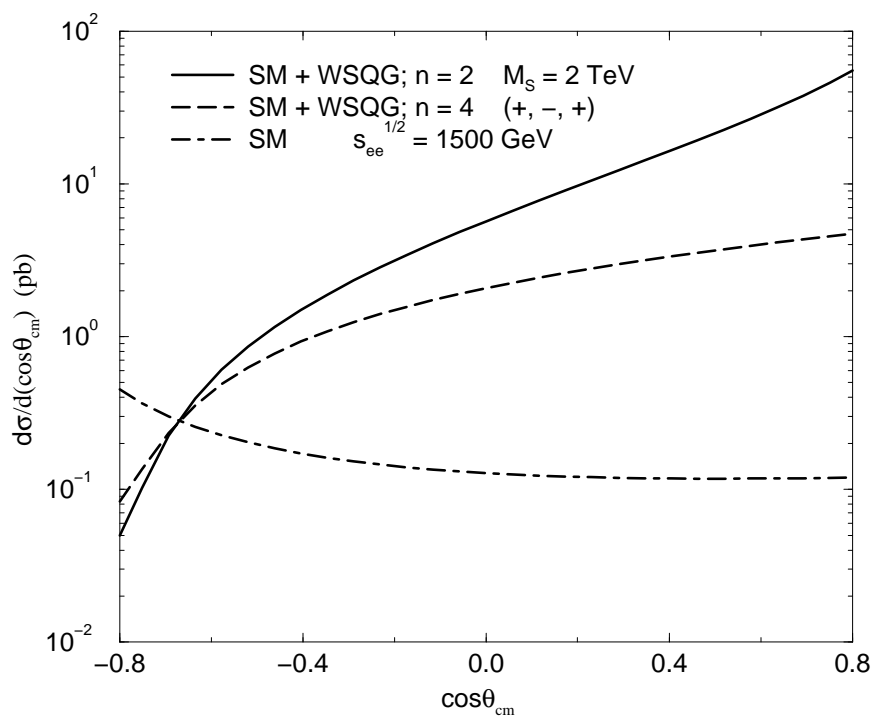

Figure 3: SM + WSQG and SM differential cross sections at $\sqrt{s_{e e}}=1500 \mathrm{GeV}$ for the $(+,-,+)$ polarization. Here, $M_{S}=2 \mathrm{TeV}$ and $n=2,4$, for the WSQG contributions. 


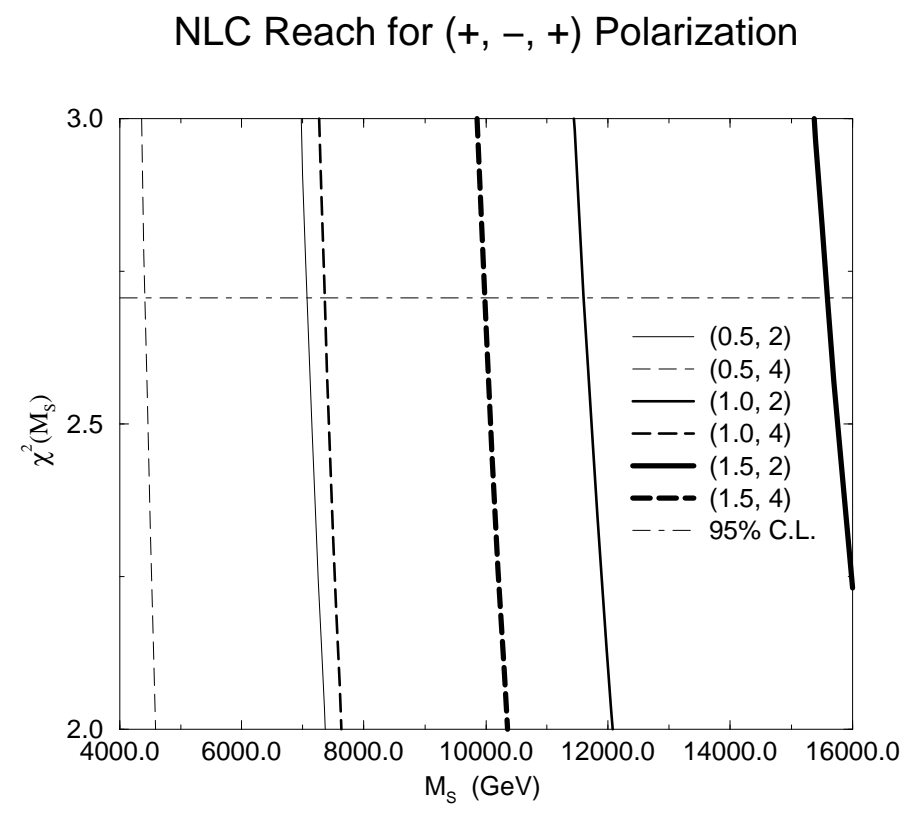

Figure 4: The solid and the dashed lines represent the $\chi^{2}$ as a function of $M_{S}$ for the cases $n=2$ and $n=4$, respectively, at three values of $\sqrt{s_{e e}}$. The numbers in the parentheses denote the value of $\sqrt{s_{e e}}$, in $\mathrm{TeV}$, and $n$, respectively. The dot-dashed line marks the reach at the $95 \%$ confidence level. 


\section{References}

[1] N. Arkani-Hamed, S. Dimopoulos, and G. Dvali, Phys. Lett. B429 (1998) 263; Phys. Rev. D59 (1999) 086004.

[2] L. Randall and R. Sundrum, hep-th/9906064; hep-ph/9905221.

[3] S. Cullen and M. Perelstein, hep-ph/9903422; L.J. Hall and D. Smith, hepph/9904267.

[4] I. Antoniadis and B. Pioline, hep-ph/9906480; D. Atwood, S. Bar-Shalom, and A. Soni, hep-ph/9906400 ; A. Pilaftsis, hep-ph/9906265 T. Banks and W. Fischler, hep-th/9906038 ; T.G. Rizzo and J.D. Wells, hep-ph/9906234; E. Halyo, hep-ph/9905577; X.-G. He, hep-ph/9905500; V. Barger, T. Han, C. Kao, and R.J. Zhang, hep-ph/9905474; T. Han, D. Rainwater, and D. Zeppenfeld, hep-ph/9905423; P. Mathews, P. Poulose, and K. Sridhar, hep-ph/9905395; I. Antoniadis, K. Benakli, and M. Quiros, hep-ph/9905311; X.-G. He, hepph/9905295; A. Donini and S. Rigolin, hep-ph/9905293; K.Y. Lee, H.S. Song, J.H. Song, C. Yu, hep-ph/9905227; M. Dine, hep-ph/9905219; K. Cheung, hep-ph/9904510; A. Perez-Lorenzana and R.N. Mohapatra, hep-ph/9904504; K. Yoshioka, hep-ph/9904433; E. Halyo, hep-ph/9904432; H. Davoudiasl, hepph/9904425; T.G. Rizzo, hep-ph/9904380; hep-ph/9903475; hep-ph/9902273; hep-ph/9901209; K.Y. Lee, H.S. Song, and J.H. Song, hep-ph/9904355; H. Goldberg, hep-ph/9904318; G. Shiu, R. Shrock, and S.-H.H. Tye, hep-ph/9904262; L. Hall and C. Kolda, hep-ph/9904236; A.K. Gupta, N.K. Mondal, and S. Raychaudhuri, hep-ph/9904234; P. Mathews, S. Raychaudhuri, and K. Sridhar, hep-ph/9904232; hep-ph/9812486; K. Cheung, hep-ph/9904266; C. Balázs, et al., hep-ph/9904220; G. Dvali and A.Yu. Smirnov hep-ph/9904211; D. Atwood, S. Bar-Shalom, A. Soni, hep-ph/9903538; K. Cheung and W.-Y. Keung, hepph/9903294; T. Banks, M. Dine, and A. Nelson, hep-th/9903019; K. Agashe and N.G. Deshpande hep-ph/9902263; E.A. Mirabelli, M. Perelstein, and M.E. Peskin, Phys. Rev. Lett. 82 (1999) 2236; J.L. Hewett, Phys. Rev. Lett. 82 (1999) 4765; I. Antoniadis, N. Arkani-Hamed, S. Dimopoulos, and G. Dvali, Phys. Lett. B436 (1998) 257; Z. Berezhiani and G. Dvali, Phys. Lett. B450 (1999) 24; S. 
Nussinov and R. Shrock, Phys. Rev. D59 (1999) 105002; K. Benakli and S. Davidson, hep-ph/9810280; K. Benakli, hep-ph/9809582;

[5] I.F. Ginzburg et al., Nucl. Instr. and Meth. 205 (1983) 47; I.F. Ginzburg et al., Nucl. Instr. and Meth. 219 (1984) 5.

[6] For a review of physics at the NLC, see H. Murayama and M.E. Peskin, Annu. Rev. Nucl. Part. Sci. 46 (1996) 533.

[7] T. Han, J.D. Lykken, R.-J. Zhang, Phys. Rev. D59 (1999) 105006.

[8] G.F. Giudice, R. Rattazzi. J.D. Wells, Nucl. Phys. B544 (1999) 3. 